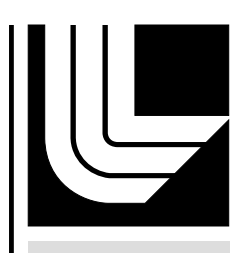

LAWRENCE LIVERMORE N A TIO NAL LABORATORY

\title{
FY2006 Engineering Tech Base Report: Defect Detection in Large CT Image Sets
}

D. N. Poland, A. Lopez, S. Manay, S. Sengupta

September 18, 2006 
This document was prepared as an account of work sponsored by an agency of the United States Government. Neither the United States Government nor the University of California nor any of their employees, makes any warranty, express or implied, or assumes any legal liability or responsibility for the accuracy, completeness, or usefulness of any information, apparatus, product, or process disclosed, or represents that its use would not infringe privately owned rights. Reference herein to any specific commercial product, process, or service by trade name, trademark, manufacturer, or otherwise, does not necessarily constitute or imply its endorsement, recommendation, or favoring by the United States Government or the University of California. The views and opinions of authors expressed herein do not necessarily state or reflect those of the United States Government or the University of California, and shall not be used for advertising or product endorsement purposes.

This work was performed under the auspices of the U.S. Department of Energy by University of California, Lawrence Livermore National Laboratory under Contract W-7405-Eng-48. 


\section{Project Overview}

This image analysis project is constructing a tool for performing computer assisted detection of defects in large CT data sets. We address two primary challenges: proving an algorithm that can reliably highlight the objects of interest, and coupling this algorithm to efficient and effective data management and interface routines.

\section{Project Goals}

The goal of this project is to create an end to end tool that will efficiently extract from large CT data sets a manageable set of candidate defects that highlights $100 \%$ of the unambiguous (to a trained analyst) true defects and as many as possible of the ambiguous objects. Objective metrics for defect ambiguity are elusive given the ill defined nature of both the defects and their CT images (e.g., variations in size and contrast, defect/object density gradients). Therefore the true metric of success will be gaining the confidence of the Weapons Program NDE analysts in this tool, which will be a qualitative function of efficiency and accuracy.

In consultation with Weapons Program NDE analysts voids it was decided to focus this initial effort on searching for small voids (order of tenths to tens of millimeters in extent). The program has produced a Tungsten ring with surrogate defects drilled into it (Figure 1) that they use for studying the ability of their systems and analysts to detect this class of defects.

\section{Relevance to LLNL Mission}

This tool will begin to make the terabyte Stockpile Surveillance data sets that will be produced in the immediate future more tractable, and provide a foundation for more robust and rigorous analysis. It will establish an architecture into which additional algorithms that highlight other classes of defects may be inserted and would apply to other CT analysis problems where such algorithms exist.

\section{FY2006 Accomplishments and Results}

The radiography and image reconstruction processes produce a wealth of data that can be viewed in many different ways. Due to the ill defined nature of the defects being sought, it is unclear how best to proceed, and in fact analysts tend to develop multi-pronged approaches using several different types of data to develop and confirm hypotheses. The radiographs themselves, one for each of several hundred angles looking through a rotating object, contain pixels which are proportional to an integral of the object density along a line drawn between the source and that pixel. In viewing these as a sequence, one can see object features moving side to side as the object rotates. One can extract a given row (elevation) from each radiograph in this sequence and stack them, creating a sinogram in which small features form a distinct sinusoid pattern as the object rotates. We looked for these sinusoid patterns using the Gradient Direction Matching (GDM) [1] algorithm developed under the Image Content Engine (ICE) LDRD-SI [2], a powerful 
model-based matching tool (Figure 2). This approach was attractive due to the success of GDM in similar problems, the usefulness of sinogram data to human analysts, and the fact that it does not require image reconstruction.

Once the CT image reconstruction is performed, one can look for defects in either $2 \mathrm{~d}$ image slices (pixels) or $3 \mathrm{~d}$ volumes (voxels). We searched for localized bright or dark regions indicative of defects in these data using mathematical morphology (MM) [3] based algorithms (Figure 3). Based on max/min operations in a pixel or voxel neighborhood, MM filters are nonlinear, and skillfully constructed can solve many problems not amenable to solution by classical linear filtering methods.

Using both simulated and real data, we implemented and evaluated these approaches. We found that the MM/CT approach is more robust for these data sets due to the low sinogram SNR associated with small defects. There is promise that GDM/sinogram analysis could be developed as a confirmatory tool for $\mathrm{MM} / \mathrm{CT}$, perhaps employing a Bayesian framework that leverages the available CT, sinogram, and design data, but this must be left for future research.

\section{FY2007 Proposed Work}

We are currently in the process of quantifying the performance of the MM algorithm using real weapon system CT data, and will then implement automated parameter selection (e.g., automated thresholding based on global and local statistics). The final steps will be end to end tool integration, and a report on future research needs.

\section{References}

[1] David W. Paglieroni, Walter G. Eppler and Douglas N. Poland, "Phase Sensitive Cueing for 3D Objects in Overhead Images", SPIE Defense and Security Symposium: Signal Processing, Sensor Fusion and Target Recognition XIV, Proc. SPIE, Vol.5809, 28-30 March 2005, Orlando FL.

[2] James M. Brase, David W. Paglieroni, Douglas N. Poland, George F. Weinert, Charles W. Grant, Aseneth S. Lopez and Sergei Nikolaev, "Image Content Engine (ICE): A System for Fast Image Database Searches", SPIE Defense and Security Symposium: Optics and Photonics in Global Homeland Security, Proc. SPIE, Vol.5781, 28-30 March 2005, Orlando, FL.

[3] J. Serra, Image Analysis and Mathematical Morphology, Academic Press, New York, 1982. 


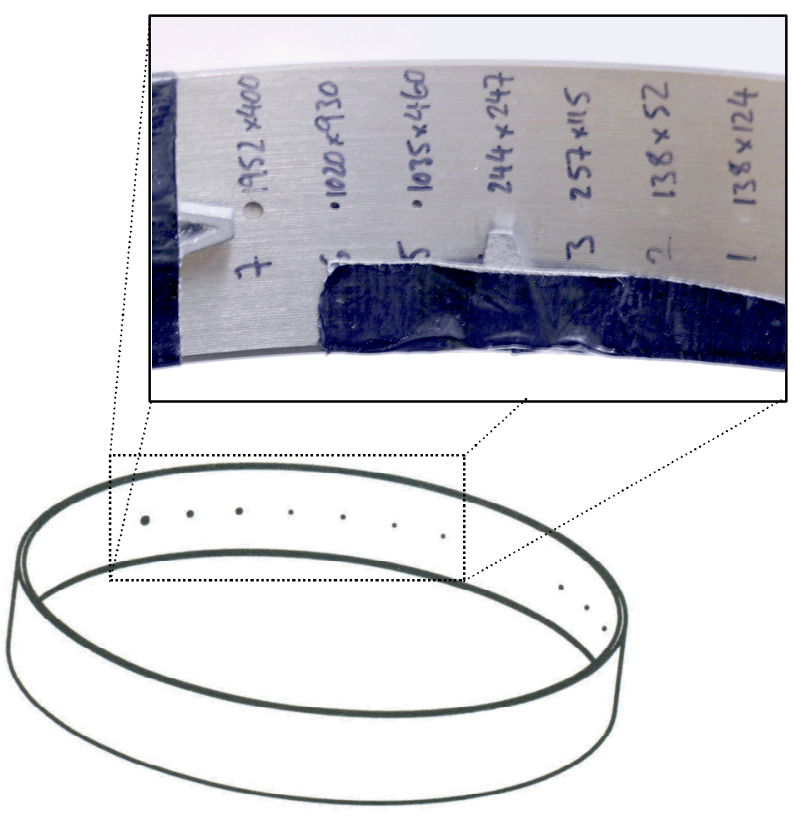

Figure 1: CAD drawing of Tungsten ring with photo of series of 7 holes 

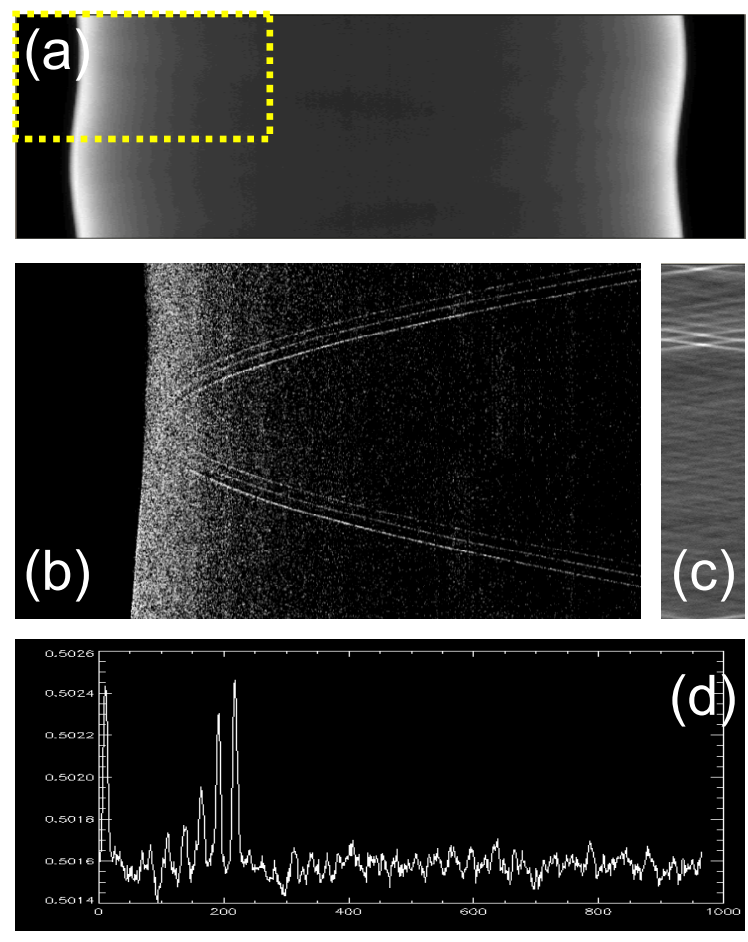

Figure 2: GDM/sinogram processing of Tungsten ring. (a) raw sinogram (stack of radiograph lineouts) at object height containing 6 holes ranging from 300 to 500 microns. (b) processed sinogram, suppressing homogeneous (intact object) signal. (c) match surface output from GDM algorithm - brightness corresponds to degree of match to sinusoid of a given phase and amplitude. (d) lineout of (c), where defects show as peaks 

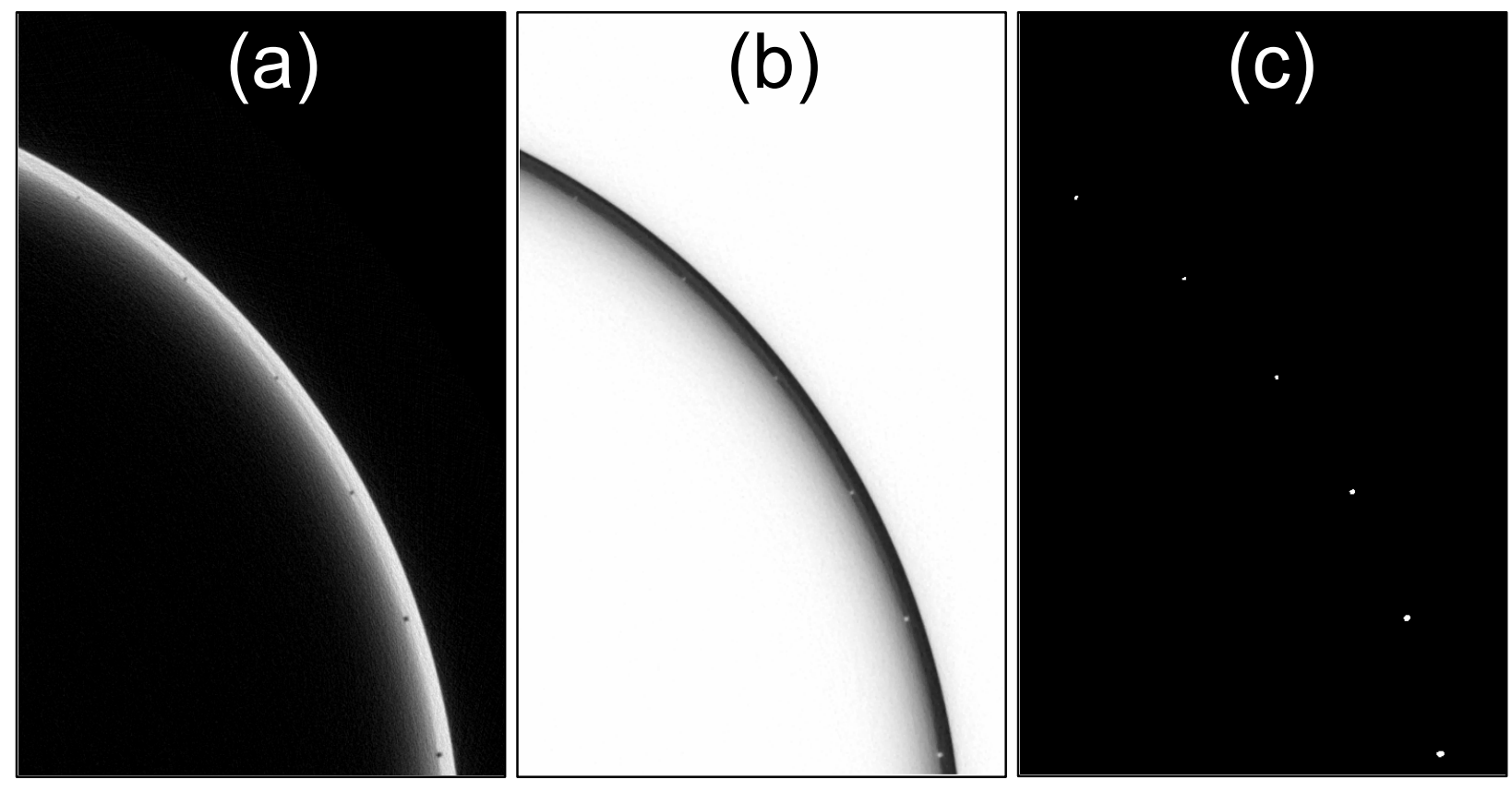

Figure 3: MM/CT processing of Tungsten ring. (a) individual reconstructed CT image slice containing 6 holes ranging from 300 to 500 microns. (b) result of applying MM alternating sequential filter and complementing the image. (c) result highlighting the "defects" after $3 \mathrm{~d}$ MM white tophat reconstruction and thresholding steps. 